\title{
A Formally Verified Decentralized Key Management Architecture for Wireless Sensor Networks
}

\author{
Yee Wei Law, Ricardo Corin, Sandro Etalle, and Pieter H. Hartel \\ Faculty of Electrical Engineering, Mathematics and Computer Science \\ University of Twente, PO Box 217, 7500 AE Enschede, The Netherlands* \\ $\{$ ywlaw, corin, etalle, pieter\}@cs . utwente.nl \\ http://eyes.eu.org
}

\begin{abstract}
We present a decentralized key management architecture for wireless sensor networks, covering the aspects of key deployment, key refreshment and key establishment. Our architecture is based on a clear set of assumptions and guidelines. Balance between security and energy consumption is achieved by partitioning a system into two interoperable security realms: the supervised realm trades off simplicity and resources for higher security whereas in the unsupervised realm the vice versa is true. Key deployment uses minimal key storage while key refreshment is based on the well-studied scheme of Abdalla et al. The keying protocols involved use only symmetric cryptography and have all been verified with our constraint solving-based protocol verification tool CoProVe.
\end{abstract}

Keywords: Wireless sensor networks, key management, protocol verification.

\section{Introduction}

Wireless sensor networks (WSNs) are open architectures, where any potential intruder can easily intercept, eavesdrop and fake messages. Therefore, to guarantee any level of security (confidentiality, authentication etc.) one has to employ cryptographic protocols.

Key management is the process by which cryptographic keys are generated, stored, protected, transferred, loaded, used, and destroyed. Key management is a challenging problem for WSNs due to the hardware constraints of the sensors and the dynamic nature of WSNs themselves. The hardware constraints of sensors are in terms of:

1. Cost: Sensors are generally not tamper-resistant.

2. Space: They can only store as many keys as are usually allowed by the storage left over by the operating system and application code, which is not much.

* This work is partially sponsored by the European Union as part of the EYES project IST-2001-34734. 
3. Energy: It is necessary to optimize the use of cryptography since cryptographic operations tend to be resource-intensive.

4. Time: Public-key cryptography should be avoided or at least limited to applications which are not time-constrained, because they are a few order of magnitude more resource and time consuming than symmetric-key cryptography [3].

The security and functional requirements of most WSNs are such that under the above constraints, the following guidelines need to be taken into account:

1. NO_SINGLE_KEY: The system should not operate on a system-wide key (or keys). Due to the sensor nodes' lack of tamper-resistance, having a vulnerable system-wide key is not better than having no key at all.

2. NO_SINGLE_POINT: The system should not have a single point (e.g. node) of failure.

3. SCALABILITY: The system should be scalable in the sense that the addition of new nodes should not cause excessive rise in computation, communication and administrative overhead in the network.

As of writing, we are not aware of any key management architecture that satisfies all the above hardware constraints as well as the guidelines. Take for example Basagni et al's proposal [2]. In this proposal, sensors are assumed to be tamper-resistant (contrary to our hardware constraints) and share a networkwide system key. Although repeated key refreshment (aka re-keying) would thwart cryptanalytic attampts on the traffic encryption key, the compromise of a single node compromises the system key and thus the whole network, leading to the violation of guideline NO_SINGLE_KEY and NO_SINGLE_POINT. In Perrig et al's proposal [12], the testbed architecture for the SPINS protocol suite possesses a single point of vulnerability that is the base station, violating guideline NO_SINGLE_POINT in addition to SCALABILITY.

The contributions of this paper are:

1. A decentralized key management architecture for WSNs, covering the aspects of key deployment, key refreshment and key establishment. Our decentralized key management architecture, which we call EYES Security Architecture 1 (ESA1), satisfies all the above hardware constraints and architecture guidelines. In addition, authenticated routing is directly implementable on top of the architecture, allowing an integrated security architecture to be specified.

2. Verification of all the protocols employed in our architecture using CoProVe [6]. The verification results imply that our protocols are secure in our standard set of test scenarios.

Overview. We start by giving the preliminaries in Section 2 , which covers the notation that we use, and the overview and security objectives of our architecture. Section 3 proceeds to describe key deployment, i.e. the strategic distribution of keys on sensor nodes during the bootstrap phase; and key refreshment, i.e. the refreshment of keys for increasing the encryption threshold. Section 4 describes keying protocols, i.e. the establishment of common session keys to secure 
communication channels; and their verification. Section 5 discusses important related work. Section 6 concludes and wraps up with a list of future work.

\section{Preliminaries}

Notation. To simplify the discussion below, we are using the following standard notation:

- $E_{K}(M)$ represents the ciphertext as a result of encrypting plaintext $M$ with key $K$.

- $M A C_{K}(M)$ represents the message authentication code (MAC) of message $M$ using key $K$.

- If $A, B \in\{0,1\}^{*}$, then $A \mid B$ is their concatenation.

We also use the following non-standard notation:

- $A \rightsquigarrow B$ : $A$ 's radio range reaches $B$, or equivalently $A$ 's transmission reaches $B$ in a single hop; but not necessarily vice versa.

$-A \rightsquigarrow B: A \rightsquigarrow B$ and $B \rightsquigarrow A$.

Architecture. In ESA1, we employ a structure of supervised and unsupervised clusters. A supervised cluster consists of a supervisor node (e.g. $S^{\alpha}$ of cluster $\alpha$, $S^{\beta}$ of cluster $\beta$ in Figure 1 (a)) and a group of supervised nodes (e.g. $N_{1}^{\alpha}$ of cluster $\alpha, N_{1}^{\beta}$ and $N_{2}^{\beta}$ of cluster $\beta$ in Figure $1(\mathrm{a})$ ). While the supervised nodes are normal sensor nodes, the supervisor is usually assumed to be tamper-resistant, to have higher energy and computational resources (like the Rich Uncles in [4]), and to have wider radio coverage compared with normal sensor nodes. An unsupervised cluster consists only of unsupervised nodes (e.g. $N_{1}^{\gamma}$ of cluster $\gamma$ in Figure 1(a)), which usually are just normal sensor nodes.

In our architecture, supervisors are needed to combine security (confidentiality and authentication) with energy efficiency and scalability. Nodes that are only sporadically involved in security-critical tasks requiring confidentiality and authentication can be left unsupervised.

Another motivation for having supervisors as specified is to support Di Pietro et al's secure selective exclusion [8] and intrusion detection, the details of which is however outside the scope of this paper. In other words, the above specification of the supervisors is not a limitation of our model. We allow as many or as few supervisors as desirable from a cost point of view.

Security Objectives. First, we define two security realms: the set of all supervised clusters constitute the supervised realm, while the set of all unsupervised clusters constitute the unsupervised realm.

- In the supervised realm, confidential and authenticated node-to-node communication within a single supervised cluster, and between nodes residing in different supervised clusters are realized through intra-supervised cluster keying (cf Section 4.2) and respectively inter-supervised cluster keying 


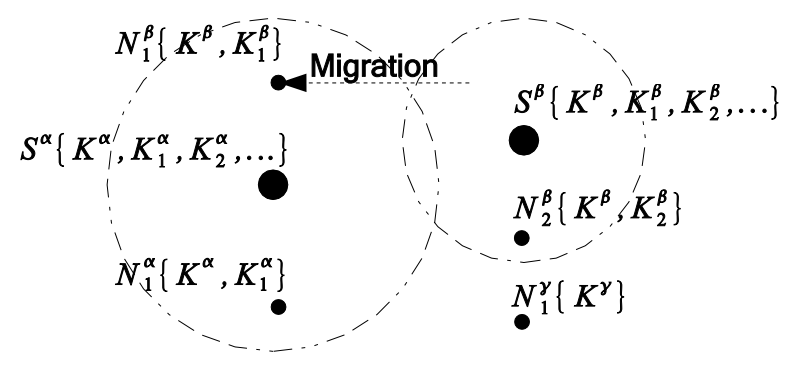

(a)

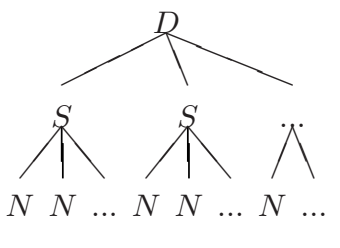

(b)

Fig. 1. (a) Key deployment in ESA1 (where $N_{i}^{\alpha}\left\{K^{\alpha}, K_{i}^{\alpha}\right\}$ means node $N_{i}^{\alpha}$ of cluster $\alpha$ with keys $K^{\alpha}, K_{i}^{\alpha}$; dashed circles represent supervisors' radio range). (b) Three-tier hierarchy for the supervised realm: $D=$ Domain, $S=$ Supervisor, $N=$ Node.

(cf Section 4.3). In these modes of communication, the effect of any node's compromise is isolated, i.e. if a supervised node $N_{1}^{\alpha}$ is compromised, only sessions that involve $N_{1}^{\alpha}$ are compromised.

- In the unsupervised realm, confidential and authenticated group communication within a single unsupervised cluster is realized through intraunsupervised cluster keying (cf Section 4.4). In this mode of communication, the compromise of a node compromises the security of the whole cluster. Between unsupervised clusters, communication is open and unauthenticated, i.e. no two unsupervised clusters trust each other.

- Between the supervised realm and the unsupervised realm, confidential and authenticated communication is realized through unsupervised-to-supervised cluster keying (cf Section 4.5). Since the supervised realm has a higher security level than the unsupervised realm, we do not think it makes sense to secure a communication that originates from a supervised node to an unsupervised node (drawing an analogy from the "no write-down" policy of the Bell LaPadula model). However the need for unsupervised-to-supervised node communication is believed to be quite possible, e.g. in the upload of sensitive data.

Independent of the confidentiality and authentication requirements, message integrity can and should always be ensured by using universal hash functions [5]. Universal hash functions are highly resistant to collisions and have also been recently shown to be resistant to algorithmic complexity-based denial of services attacks [7.

\section{Key Deployment}

We can now describe the main keys employed in ESA1 and their roles.

In ESA1, every supervised node $N_{i}^{\alpha}(1 \leq i \leq n)$ of cluster $\alpha$ is equipped with a unique node key $K_{i}^{\alpha}$ and the supervisor $S^{\alpha}$ is equipped with the node keys of 
all the supervised nodes of its cluster. Additionally $S^{\alpha}, N_{1}^{\alpha}, \ldots, N_{n}^{\alpha}$ share the same cluster key $K^{\alpha}$. The cluster key is a backup key that is only used when the supervisor is not available, in which case the supervised cluster downgrades to an unsupervised cluster. Therefore in contrast to what Figure 1(a) might suggest, the cluster membership of a node $N$ in cluster $\alpha$ depends solely on (1) whether there is a unique shared key between $N$ and $S^{\alpha}$; and (2) whether $N$ possesses the cluster key $K^{\alpha}$. When a node, say $N_{1}^{\beta}$ (cf Figure 1(a)), roams out of the radio range of its original supervisor $S^{\beta}$, to the radio range of another supervisor $S^{\alpha}$, it will for efficiency initiate a process called migration that migrates its node key from $S^{\beta}$ to $S^{\alpha}$ (cf Section 4.6). It is important to note that when we mention "migrating a key", we actually mean "migrating a derivation of the key", so that even if the migrated key is compromised backward secrecy is preserved.

In an unsupervised cluster $\gamma$, every node shares the same cluster key $K^{\gamma}$, and cluster membership depends solely on the possession of $K^{\gamma}$.

To establish trust between supervisors, we leave two options open. The first option involves a further domain tier higher in the hierarchy (cf Figure 1(b)), and it is to connect all the supervisors at bootstrap to a domain controller. The domain controller partitions the set of supervisors into domains. Only supervisors that belong to the same domain are given the same domain key (a symmetric key) and thus share the common trust. This scheme uses only symmetric cryptography but can only support a static and coarse notion of trust - the compromise of any supervisor compromises the whole domain. The second option is to resort to SSL/TLS (or similar public key-based mechanisms) to secure communication between supervisors. This scheme supports flexible and fine-grained access control at the expense of higher overhead and energy consumption. All in all, the suitability of each of these two options depends on the construction of the supervisors and the actual application scenario. In this paper, we focus on the node/cluster tiers of the hierarchy.

\subsection{Key Refreshment}

The effectiveness of a key is not only bounded by its length but also by the number of encryptions it has been used for, because with enough encryptions an intruder is able to launch birthday attacks (or cryptanalytic attacks which however typically require more encryptions [1]). Therefore, as part of any key management architecture, we need a scheme to refresh every now and then the deployed keys. For this, we refer to the well-studied scheme of Abdalla et al [1]. Given an initial shared key $K_{0}$ between two nodes $A$ and $B$, the encryption and message authentication code (MAC) keys are derived serially at each $i$-th re-key as follows:

$$
K_{e n c, i}=M A C_{K_{i}}(1) \quad \text { and } \quad K_{M A C, i}=M A C_{K_{i}}(2)
$$

where $K_{i+1}=M A C_{K_{i}}(0)$ and $i=0,1,2, \ldots$ A convention in this paper is that a pair of encryption key and MAC key derived from key $K$ is written as $K^{\prime}$ and $K^{\prime \prime}$. 
In a supervised cluster, re-keying is message-count driven, i.e. when the encrypted message count approaches the encryption threshold (e.g. $2^{2 k / 3}$ for CBC mode, where $k$ is the key length [1]), re-keying is initiated. In a supervised cluster, if the shared key is between a node and the superviser, or between a node and another node, re-keying can be initiated by either end of the secure channel. In an unsupervised cluster, re-keying of the cluster key can proceed in a similar fashion described by Basagni et al [2].

\section{$4 \quad$ Verified Keying}

In our end-to-end communication model, session establishment has two phases:

1. Keying: The initiator engages the node it wants to communicate with in a key establishment (keying for short) protocol, using an underlying, not necessarily secure routing protocol.

2. Communication: Then, by using the session key established, the two endpoints start communicating with authenticated and optionally encrypted messages.

The following discusses the keying protocols and their formal verification.

\subsection{Formal Protocol Verification}

The keying protocols described in this section are verified using the protocol verifier CoProVe (available at http: //wwwes.cs. utwente.nl/24cqet/coprove.html [6]). The verifier works by taking as input a specification of the protocol and a system scenario describing the roles involved in the protocol, e.g. the initiator, the responder or a server of the protocol. The system scenario is analyzed by the verifier in search of possible interleavings of the roles. Attacks manifest as interleavings not anticipated by the protocol designer.

In this paper, CoProVe is used to verify these properties:

- Confidentiality: A session key must only be known to the communicating nodes, and the supervisors involved in the keying protocol.

- Authentication: A keying protocol must end with every party properly authenticating the other parties it is communicating with. In other words, it must be impossible for any intruder $I$ to impersonate another node $A$ whose keys (used in the keying protocol) $I$ does not have.

- Replay resistance: The meaning of replay attack on a role $R$ is the possibility of unauthenticated parties to cause $R$ to run, i.e. for $R$ to process replayed messages. If $R$ happens to maintain state of every run, then it would be maintaining incorrect states. In CoProVe, we verify replay resistance of $R$ by making sure that two instances of $R$ cannot complete regardless of the other roles. 
Limitations. To ensure termination, system scenarios must be finite - a restriction shared by every model-checking-based approach. (Other approaches like theorem proving allow an unbounded number of sessions, but sacrifice termination guarantee, or need human intervention.) As such, a protocol is verified secure only in the finite tested scenarios. There is no assurance that an attack would not actually be found in a larger scenarios.

So far, we have tested all protocols in the context of a scenario containing a single session. Such tests turned out to be extremely useful, as they have allowed us for example to spot authentication failures resulting from the inappropriate use of unauthenticated nonces. Here, we also want to stress that these protocols are considerably more complex than many of the usual "toy" security protocols protocol verifiers are tested agains. In fact, considering that one of them involves nine message exchanges, we were ourselves pleasantly surprised to see that CoProVe could deal with them within a reasonable time-span (ranging from a fraction of a second to 2.5 days for the most complex protocol, on a standard Linux-i686 architecture). We are now carrying out extra tests involving larger scenarios.

\subsection{Intra-supervised Cluster Keying}

We start with the keying protocol that is used within a supervised cluster. Concerning the notation, $N_{A}$ represents a nonce emitted by $A, N_{B}$ represents a nonce emitted by $B$ and so on; strings such as Ack and Success are constants. For the sake of notation simplicity, we leave out the superscripts we used in Figure 1 In addition, recall that the encryption key and the MAC key derived from key $K$ are respectively denoted by $K^{\prime}$ and $K^{\prime \prime}$.

Suppose that in a cluster supervised by $S$, a node $A$ (which shares key $K_{A}$ with $S$ ) wants to initiate a session with $B$ (which shares key $K_{B}$ with $S$ ). The protocol is:

\section{Protocol 1}

1. $A \rightarrow S: N_{A}, B, \mathrm{MAC}_{K_{A}^{\prime \prime}}\left(N_{A} \mid B\right)$

2. $S \rightarrow A: E_{K_{A}^{\prime}}\left(E_{K_{B}^{\prime}}\left(N_{S}\right) \mid K_{A B}\right), \operatorname{MAC}_{K_{A}^{\prime \prime}}\left(N_{A}|B| E_{K_{B}^{\prime}}\left(N_{S}\right) \mid K_{A B}\right)$

3. $A \rightarrow B: A, E_{K_{B}^{\prime}}\left(N_{S}\right)$

4. $B \rightarrow S: B, N_{B}, A, \mathrm{MAC}_{K_{B}^{\prime \prime}}\left(N_{S}|B| N_{B} \mid A\right)$

5. $S \rightarrow B: E_{K_{B}^{\prime}}\left(K_{A B}\right), \mathrm{MAC}_{K_{B}^{\prime \prime}}\left(N_{B}|A| E_{K_{B}^{\prime}}\left(K_{A B}\right)\right)$

6. $B \rightarrow A: A c k, \mathrm{MAC}_{K_{A B}^{\prime \prime}}(A c k)$

where $K_{A B}$ is the final established session key. This protocol has been verified (1) secure in the confidentiality of $K_{A B},(2)$ secure in the mutual authentication between $A$ and $B$, and (3) secure against replay attacks on $S$. Before we proceed to describe this protocol, we would like to state that an alternative keying protocol could have been derived from the node-to-node key agreement protocol of SPINS [12]: 


\section{Protocol 2}

$$
\begin{aligned}
& \text { 1. } A \rightarrow B: N_{A}, A \\
& \text { 2. } B \rightarrow S: N_{A}, N_{B}, A, B, \operatorname{MAC}_{K_{B}^{\prime \prime}}\left(N_{A}\left|N_{B}\right| A \mid B\right) \\
& \text { 3. } S \rightarrow A: E_{K_{A}^{\prime}}\left(K_{A B}\right), \operatorname{MAC}_{K_{A}^{\prime \prime}}\left(N_{A}|B| E_{K_{A}^{\prime}}\left(K_{A B}\right)\right) \\
& \text { 4. } S \rightarrow B: E_{K_{B}^{\prime}}\left(K_{A B}\right), \operatorname{MAC}_{K_{B}^{\prime \prime}}\left(N_{B}|A| E_{K_{B}^{\prime}}\left(K_{A B}\right)\right) \\
& \text { 5. } A \rightarrow B: A c k, \operatorname{MAC}_{K_{A B}^{\prime \prime}}(A c k)
\end{aligned}
$$

Here, the first four messages are from SPINS and we added the fifth message to prevent authentication failure. This protocol has been verified (1) secure in the confidentiality of $K_{A B}$, (2) secure in the mutual authentication between $A$ and $B$, and (3) secure against replay attacks on $S$. The drawback of this protocol is that while there is no attack, it is far too easy for an intruder to generate random bogus requests (at step 1) and hence random bogus protocol instances, that would cause $B$ to wait at step 5 for the bogus protocol instances to finish.

A design principle behind Protocol 1 is that $A$ should do more work compared with $B$ to initiate the protocol. Now it takes three messages for $A$ to get $B$ to respond, and $S$ is able tell $B$ whether $A$ 's request is genuine at step 5. Let's also have a look at the energy budget. We only consider the energy consumed in radio communication, since it generally dominates that used for computation. Assuming the following sizes: node names (IDs) and nonces are 64 bits long, MACs are 160 bits, keys are 128 bits, acknowledgements are 8 bits (to cater for other status codes), encryption is length-preserving; and assuming the energy consumption for receiving to be $40 \%$ that of sending 13, we found the energy consumption of Protocol 1 compared with Protocol[2] to be $52 \%$ higher for $A, 6 \%$ for $B$ and $21 \%$ for $S$. The fact that $A$ has to consume more energy in Protocol 1 cannot be helped by our requirement that $A$ authenticates itself with $S$ first, but this is necessary to overcome the drawback of Protocol 2 Moreover, the energy cost can be amortized over $A$ 's period of operation, provided such keying is conducted infrequently.

Special attention is given to the MAC at step 6 of Protocol1 1 because MAC'ed acknowledgements are susceptible to replays depending on the MAC mode used. By assuming that combined CTR and CBC mode (CTR + CBC-MAC) [10] is used, we should be able to minimize this risk. This also applies to other instances of MAC'ed acknowledgement in the other protocols in the rest of this paper.

\subsection{Inter-supervised Cluster Keying}

After solving the problem of keying two nodes in the same cluster, let us consider keying two nodes from two different clusters in the supervised realm. The protocol is: 


\section{Protocol 3}

1. $A \rightarrow S_{A}: N_{A}, B, \mathrm{MAC}_{K_{A}^{\prime \prime}}\left(N_{A} \mid B\right)$

2. $S_{A} \rightarrow A: E_{K_{S S}^{\prime}}\left(N_{S_{A}}\right), \operatorname{MAC}_{K_{A}^{\prime \prime}}\left(N_{A}|B| E_{K_{S S}^{\prime}}\left(N_{S_{A}}\right)\right)$

3. $A \rightarrow B \quad: A, B, E_{K_{S S}^{\prime}}\left(N_{S_{A}}\right)$

4. $B \rightarrow S_{B}: B, N_{B}, A, E_{K_{S S}^{\prime}}\left(N_{S_{A}}\right), \mathrm{MAC}_{K_{B}^{\prime \prime}}\left(B\left|N_{B}\right| A \mid E_{K_{S S}^{\prime}}\left(N_{S_{A}}\right)\right)$

5. $S_{B} \rightarrow S_{A}: N_{S_{B}}, A, B, E_{K_{S S}^{\prime}}\left(K_{A B 2}\right), \operatorname{MAC}_{K_{S S}^{\prime \prime}}\left(N_{S_{A}}\left|N_{S_{B}}\right| A|B| E_{K_{S S}^{\prime}}\left(K_{A B 2}\right)\right)$

6. $S_{A} \rightarrow S_{B}: E_{K_{S S}^{\prime}}\left(K_{A B 1}\right), \operatorname{MAC}_{K_{S S}^{\prime \prime}}\left(N_{S_{B}}|A| B \mid E_{K^{\prime \prime} S S}\left(K_{A B 1}\right)\right)$

7. $S_{A} \rightarrow A: E_{K_{A}^{\prime}}\left(K_{A B}\right), \mathrm{MAC}_{K_{A}^{\prime \prime}}\left(N_{A}|B| E_{K_{A}^{\prime}}\left(K_{A B}\right)\right)$

8. $S_{B} \rightarrow B: E_{K_{B}^{\prime}}\left(K_{A B}\right), \operatorname{MAC}_{K_{B}^{\prime \prime}}\left(N_{B}|A| E_{K_{B}^{\prime}}\left(K_{A B}\right)\right)$

9. $B \rightarrow A \quad: A c k, \mathrm{MAC}_{K_{A B}}(A c k)$

where $K_{S S}$ is intuitively the shared key between $S_{A}$ and $S_{B}$; and $K_{A B}=$ $K_{A B 1} \mid K_{A B 2}$ is the final established session key. To divide the cost of generating the whole key, the key is generated as a concatenation of two sub-keys $K_{A B 1}, K_{A B 2}$ respectively produced by $S_{A}$ and $S_{B}$. Note that $A$ does not need to know who $B$ 's supervisor is - that is $S_{A}$ 's (and $B$ 's) job. This protocol has been verified (1) secure in the confidentiality of $K_{A B},(2)$ secure in the mutual authentication between $A$ and $B$, and (3) secure against replay attacks on $S_{A}$ and $S_{B}$.

\subsection{Intra-unsupervised Cluster Keying}

When two nodes in an unsupervised cluster want to communicate with each other, the only key they can use is the cluster key. To state the obvious, the cluster key is never used directly, instead, for each session a new pair of encryption and MAC keys are derived from the cluster key using the mechanism of Section 3.1.

\subsection{Unsupervised-to-Supervised Node Keying}

As mentioned, there is by default no trust between any supervised cluster and unsupervised cluster, i.e. there is no direct shared key an unsupervised node can use to communicate with a supervised node. It is here that the idea of visitor certificate is employed. A visitor certificate takes the form $E_{K_{g}}\left(I D \mid K_{v}\right)$, where $K_{g}$ is the grant key, $K_{v}$ is the visitor key and $I D$ is the visitor's name. $K_{g}$ is held by a supervisor while $K_{v}$ and the respective visitor certificate are held by an unsupervised node.

To start a session, the unsupervised node, say $U$ (with visitor key $K_{v}$ ), submits its visitor certificate $E_{K_{g}}\left(U \mid K_{v}\right)$ to the supervisor, say $S$ (with grant key $\left.K_{g}\right)$. Upon reception, $S$ decrypts $U$ 's certificate with $K_{g}$ to obtain $K_{v}$, and sends a new session key $K_{U S}$ to $U$, thereby establishing a secure session with $U$ : 


\section{Protocol 4}

$$
\begin{aligned}
& \text { 1. } U \rightarrow S: N_{U}, S, E_{K_{g}}\left(U \mid K_{v}\right), \operatorname{MAC}_{K_{v}^{\prime \prime}}\left(N_{U}|S| E_{K_{g}}\left(U \mid K_{v}\right)\right) \\
& \text { 2. } S \rightarrow U: N_{S}, E_{K_{v}^{\prime}}\left(K_{U S}\right), \operatorname{MAC}_{K_{v}^{\prime \prime}}\left(N_{U}\left|N_{S}\right| E_{K_{v}^{\prime}}\left(K_{U S}\right)\right) \\
& \text { 3. } U \rightarrow S: A c k, \operatorname{MAC}_{K_{U S}^{\prime \prime}}\left(N_{S} \mid A c k\right)
\end{aligned}
$$

Protocol 4 has been verified (1) secure in the secrecy of $K_{U S}$, and (2) secure in the mutual authentication between $U$ and $S$. From this point onwards, the keying protocol for the communication between $U$ and any node supervised by $S$, let's say $A$ will be similar to the intra-supervised cluster keying between $U$ and $A$ (cf Section 4.2).

The merit of this approach is that $K_{g}$ and $K_{v}$ can be pre-deployed, while the visitor certificate can be generated and distributed at runtime by a visit granting agent (VGA). A VGA keeps an application-determined set of visitor and grant keys. Under guideline NO_SINGLE_POINT (cf Section 1), no single VGA should possess all the visitor and grant keys of the whole network, therefore there should be several VGAs throughout the network, each safeguarding a preferrably non-intersecting set of visitor and grant keys. A VGA works with supervisors. Upon an unsupervised node $U$ 's request for a visitor certificate to visit supervisor $S$, a VGA

1. checks with $S$ and $U$ 's neighbouring supervisors if $U$ is a green node (i.e. a good node 8])

2. checks with itself if it has $U$ 's $K_{v}$ and $S^{\prime}$ s $K_{g}$

If all checks are positive, then the VGA will proceed to issue the requested visitor certificate to $A$. Otherwise the request would be denied. In this scheme, the only pre-deployed information is the visitor keys on the unsupervised nodes and grant keys on the supervisors, instead of $(x=$ number of supervisors $\times$ number of unsupervised nodes) key pairs. Note that it is straightforward to extend the visitor certificate to include the expiry time of the certificate.

\subsection{Node Migration}

Intra-supervised cluster keying and inter-supervised cluster keying work most efficiently when $S_{A} \rightsquigarrow A$. If it happens that $S_{A} \ngtr \rightarrow A$ as a result of node mobility or other factors that break the radio link, $A$ may 'employ' another supervisor, say $S_{B}$ as its temporary supervisor if $S_{B} \rightsquigarrow A$, by migrating its node key from $S_{A}$ to $S_{B}$, provided $S_{A}$ and $S_{B}$ have mutual trust. If $A$ is not able to find such a supervisor, then no migration takes place. Note that $A$ never becomes a member of $S_{B}$ 's cluster (which implies receiving the cluster key from $S_{B}$ ), because then a compromised node would be able to roam to every cluster and collect their cluster key.

Suppose $A$ migrates from the cluster supervised by $S_{A}$ to the cluster supervised by $S_{B}$, the security objective of this protocol is to prevent unauthorized migrations, i.e. if $A$ did not authorize the migration, the migration should not occur. The protocol is: 


\section{Protocol 5}

$$
\begin{aligned}
& \text { 1. } A \rightarrow S_{A}: N_{A}, S_{B}, \operatorname{MAC}_{K_{A}^{\prime \prime}}\left(N_{A} \mid S_{B}\right) \\
& \text { 2. } S_{A} \rightarrow A: E_{K_{A}^{\prime}}\left(E_{K_{S S}^{\prime}}\left(N_{S_{A}}\right)\right), \mathrm{MAC}_{K_{A}^{\prime \prime}}\left(N_{A}\left|S_{B}\right| E_{K_{S S}^{\prime}}\left(N_{S_{A}}\right)\right) \\
& \text { 3. } A \rightarrow S_{B}: A, S_{A}, E_{K_{S S}^{\prime}}\left(N_{S_{A}}\right) \\
& \text { 4. } S_{B} \rightarrow S_{A}: S_{B}, N_{S_{B}}, A, \mathrm{MAC}_{K_{S S}^{\prime \prime}}\left(N_{S_{A}}\left|S_{B}\right| N_{S_{B}} \mid A\right) \\
& \text { 5. } S_{A} \rightarrow S_{B}: E_{K_{S S}^{\prime \prime}}\left(K_{A 2}\right), \operatorname{MAC}_{K_{S S}^{\prime \prime}}\left(N_{S_{B}}|A| E_{K_{S S}^{\prime \prime}}\left(K_{A 2}\right)\right) \\
& \text { 6. } S_{B} \rightarrow A: \text { Sucess, } \mathrm{MAC}_{K_{A 2}^{\prime \prime}}(\text { Sucess })
\end{aligned}
$$

where $K_{A 2}=M A C_{K_{A}}(0)$ is the migrated key. Note that as mentioned, it is a derivation of $A$ 's node key that is migrated. This protocol has been verified (1) secure in the confidentiality of $K_{A 2}$, (2) secure in the authentication of $A$ to $S_{A}$ and $S_{B}$, of $S_{A}$ to $A$ and $S_{B}$, of $S_{B}$ to $A$ and $S_{B}$, and (3) secure against replay attacks on $S_{A}$.

Suppose $A$ migrates to $S_{C}$ 's cluster, if $S_{A}, S_{B}$ and $S_{C}$ trust each other, but $S_{A} \not \rightarrow S_{C}$ and $S_{B} \rightsquigarrow S_{C}$, then $S_{B}$ should for efficiency take over $S_{A}$ 's role of migrating $A$ 's key to $S_{C}$.

Supervisors like $S_{B}$ and $S_{C}$ are not intended to keep $A$ 's key when $A$ leaves their cluster, however if $A$ consistently returns, then it would not be efficient to purge $A$ 's key immediately once $A$ leaves. It is best to have a mechanism for predicting $A$ 's movement so that $A$ 's key can be optimally cached, otherwise there should at least be a hysteresis period where $A$ 's key is cached. Note that $S_{A}$ never purges $A$ 's key. This is to ensure that there is always one supervisor holding $A$ 's key.

\section{Related Work}

There is currently a lack of literature detailing key management architectures for WSNs. We mentioned Basagni et al's pebblenets and Perrig et al's SPINS in Section 1. We have also made a comparison between our intra-supervised cluster keying protocol with SPINS' node-to-node key agreement protocol in Section 4.2. Now we look at some other proposals.

Slijepcevic et al propose location-based keys [14. All nodes start out sharing a set of master keys, and the network is divided into non-overlapping hexagonal cells. At runtime, a node selects and activates a key from the set of master keys based on the output of its pseudorandom generator and on which hexagonal cell the node is in, so that the compromise of a key in one cell does not affect the other cells. Seen another way, the problem of session establishment reduces to the problem of determining the location; and node mobility in this scheme does not necessitate the equivalent of migration in our proposal, because it is readily 'solved' by having all nodes sharing the same keys. However, this scheme does not fit our requirements, because of our assumption that sensor nodes are not tamper-resistant - the set of master keys, the corresponding pseudorandom number generator and the seed are easily exposed and hence the active key of 
any cell can be found out. The requirement of nodes being able to discover their coordinates is also too demanding for our case.

Luo et al's localized trust model [11] is not exactly a key management architecture proposal, but it can be regarded as such. This model is based on $(k, n)$-threshold secret sharing, wherein a system secret key (of a public-private key pair) is shared among every $n$ nodes, and a node is only trusted if it acquires a certificate issued by $k$ neighbours. Session establishment is presumed to proceed through Diffie-Hellman-derived protocols. This solution forgoes the need for imposing structure (e.g. clustering) on the network, but it also means that if a node cannot get certified by $k$ neighbours, it would have to roam to another place where it can. More importantly, the extensive use of public-key cryptography is also too demanding within the resource constraints of WSNs.

\section{Conclusion and Future Work}

In this paper we have presented a decentralized key management architecture for WSNs, covering the aspects of key deployment, key refreshment and key establishment.

Motivated by the assumption that sensors are not tamper-resistant, we have devised an architecture in which two security realms namely the supervised realm and the unsupervised realm provide two levels of security. Communication in the supervised realm entails various protocols to implement intra- and intersupervised cluster keying, which we have verified with our tool CoProVe. In the unsupervsied realm, security suffers from the fact that a cluster key is shared by all members of the same cluster. The trade-off provides WSN implementors with an option to partition their system according to the actual security requirements, and the network can be expanded by easily adding one node at a time, one cluster at a time, or even one domain at a time. To conclude, our architecture meets our hardware constraints, and the guidelines NO_SINGLE_KEY, NO_SINGLE_POINT and SCALABILITY of Section 1 .

We have adopted a two-step design process by getting the protocols right first before proceeding to optimize them. Our next step is to validate our model through simulation and implementation. We are also motivated to extend our architecture to support directed diffusion (which implies multicast communication) [9]. As of now, our assumption is that a node knows exactly the name of the other node it wants to communicate with, which is not the case in directed diffusion.

Acknowledgement. The authors would like to thank Roberto di Pietro, Paul Havinga and the anonymous reviewers for their insightful comments. 


\section{References}

1. M. Abdalla and M. Bellare. Increasing the lifetime of a key: A comparitive analysis of the security of rekeying techniques. In T. Okamoto, editor, Advances in Cryptology - ASIACRYPT 2000, volume 1976 of LNCS, pages 546-565. Springer-Verlag, 2000.

2. S. Basagni, K. Herrin, D. Bruschi, and E. Rosti. Secure pebblenets. In Proceedings of the 2001 ACM Int. Symp. on Mobile Ad Hoc Networking and Computing, pages 156-163. ACM Press, October 2001.

3. M. Brown, D. Cheung, D. Hankerson, J. L. Hernandez, M. Kirkup, and A. Menezes. PGP in Constrained Wireless Devices. In 9th USENIX Security Symposium, pages 247-261. USENIX Association, Aug 2000.

4. D.W. Carman, P.S. Kruus, and B.J. Matt. Constraints and approaches for distributed sensor network security. Technical Report \#00-010, NAI Labs, 2000.

5. J.L. Carter and M.N. Wegman. Universal classes of hash functions. Journal of Computer and System Sciences, 18:143-154, 1979.

6. R. Corin and S. Etalle. An improved constraint-based system for the verification of security protocols. In M. Hermenegildo and G. Puebla, editors, 9th Int. Static Analysis Symp. (SAS), volume 2477, pages 326-341, Madrid, Spain, Sep 2002. Springer-Verlag.

7. S.A. Crosby and D.S. Wallach. Denial of service via algorithmic complexity attacks. In 12th USENIX Security Symposium. USENIX Association, 2003. To appear.

8. R. di Pietro, L.V. Mancini, and S. Jajodia. Secure selective exclusion in ad hoc wireless network. In M.A. Ghonaimy, M.T. El-Hadidi, and H.K. Aslan, editors, Security in the Information Society: Visions and Perspectives, pages 423-434. Kluwer Academic Publishers, 2002.

9. C. Intanagonwiwat, R. Govindan, and D. Estrin. Directed diffusion: A scalable and robust communication paradigm for sensor networks. In 6th Annual Int. Conf. on Mobile Computing and Networking (MobiCOM '00), pages 56-67, Boston, Massachusetts, United States, 2000. ACM Press.

10. J. Jonsson. On the security of CTR + CBC MAC. In K. Nyberg and H.Heys, editors, Selected Areas in Cryptography, 9th Annual Workshop (SAC 2002), volume 2595 of LNCS, pages 76-93. Springer-Verlag, 2002.

11. H. Luo, P. Zerfos, J. Kong, S. Lu, and L. Zhang. Self-securing ad hoc wireless networks. In 7th IEEE Symp. on Computers and Communications, pages 567-574, 2002.

12. A. Perrig, R. Szewczyk, V. Wen, D. Culler, and J.D. Tygar. SPINS: Security Protocols for Sensor Networks. In Proceedings of the 7th Ann. Int. Conf. on Mobile Computing and Networking, pages 189-199. ACM Press, 2001.

13. RF Monolithics, Inc. TR1001: 868.35 MHz Transceiver. Datasheet. http://www.rfm.com/products/data/tr1001.pdf

14. S. Slijepcevic, V. Tsiatsis, S. Zimbeck, M.B. Srivastava, and M. Potkonjak. On communication security in wireless ad-hoc sensor networks. In 11th IEEE Int. Workshops on Enabling Technologies: Infrastructure for Collaborative Enterprises, pages 139-144, Jun 2002. 